\title{
Fixture Design with FIXES: the Automatic Selection of Positioning, Clamping and Support Features for Prismatic Parts
}

\author{
J. R. Boerma, H. J. J. Kals (1); Laboratory of Production Engineering, University of Twente \\ Received on January 17, 1989
}

\begin{abstract}
FIXES is a computer aided system for the automatic generation of set-ups and for fixture design for prismatic parts. to be used in an integrated process planning ensironment. The generation of set-ups having been described in a previous paper [9], this paper concentrates on fixture design. in particular the automatic selection of the faces for the positioning, clamping and support of workpieces. The selection procedures described are based on both the topology of the prismatic part and the geometric relations between the different part elements (features). The geometric relations are evaluated with the aid of a so-called Converted Tolerance Scheme.
\end{abstract}

KEYWORDS: jigs \& fixures, set-up, posirioning, design, process plamming. CAPP, CAM

\section{Introduction}

1.1. flexibility of process planning in small batch manufacturing At present, in part manufacturing three trends can be distinguished: 1. an increase in product mix

2. an increase in the complexity of parts, due to an ongoing integration of physical functions in one part

3. an increase in accuracy, caused by miniaturization and the increased requirements for (autonated) assembly.

These factors require faster, more accurate and more flexible process planning. This concerns in particular the process of fixturing. Up till now, this task is performed in a traditional wav both in the planning phase and in the execution phase on the shop floor. A significant improvement in process planning can be achieved if the available fixturing knowledge is made explicit and accessible for process planners. They have to work according to pre-defined methods, which can be adjusted to the specific conditions in a company. In this way. the unstructured way of working with which process planning and fixturing is carried out nowadays and which prevent an effective production control, can be avoided. The use of computer equipment and dedicated software can also improve the efficiency of process planning, as it requires a formalization of the planning ciency of process planning, as it requires a forma
procedures and an inventory of the data flow.

1.2. integrated process planning

Process planning incorporates the taking of many decisions, based on workpiece data, manufacturing methods and available equipment. Process planning is concerned with decisions concerning the selection of machine planning is concerned with decisions concerning the selection of machine
tools, machining methods, cutting tools, fixtures, and the generation of cutting conditions, tool paths, NC files and instruction sheets. CAM systems are commercially available but cover only a small part of proces planning i.e. these systems are in fact no more than user-friendly NC file generating systems, in which the selection of set-ups, cutting conditions, etc. still has to be carried out by the process planner himself. Since many of the described tasks of process planning are interdependent, in future all these tasks have to be incorporated in integrated Computer Aided Process Planning (CAPP) systems. Another consideration for integration is the use of a uniform data structure to ensure the consistency and accesis the use of a uniform data structure to ensure the consistency and acces-
sibility of data. Such CAPP systems are not yet commercially available. 1.3. the PART system

An example of an integrated process planning system currently under development in our laboratory, is PART [10]; see diagram, fig. 1. This CAPP system contains six modules, each of which represents a basic step in process planning

- machine tool selection (MTS)

- selection of jigs \& fixtures (J\&F)

- determination of machining methods and sequences (MM)

- delection of cutting tools (TS)

- calculation of cutting conditions (CC)

- capacity planning (PL)

All the data needed and generated by the different modules are stored in a common database. Interaction with the system as a whole is possible via a supervisor by means of a general user interface.

Input of product models or the creation of them is possible using a boundary representation solid modeller (GPM). Output to the machining equipment is provided for by the NC module.

The six main modules are principally executed on a parallel basis. The The six main modules are principally executed on a paralle! basis. The modules in accordance with pre-defined scenarios; through the selection of a scenario out of a collection of scenarios. the process planning procedure can be adapted to a preferential planning sequence.

The FIXES system described in this paper, forms an integrated part of the $\mathrm{J} \& \mathrm{~F}$ module. The system includes two functional modules supporting the selection of set-ups and the design of fixtures. The set-up selection procedure has been reported previously [9]. The second module, including cedure has been reported previously [9]. The second module, including the selection of posit
ject of this paper.

The PART prototype system is being developed in a four-year project and sponsored by the Dutch Government as one of the hev projects in the national program for stimulation of computer science (SPIN). The prototspe will be ready by the end of 1990

\section{Present positioning methods and problems}

2.1. the need for an exact position and orientation of parts The process of fixturing consists of at least two, geographically separated phases: (i) the planning of fixtures in the process planning department and (ii) the application of fixture tools on the shop floor.

The first phase includes the selection of set-ups and the design of appropriate fixtures. The latter deals with the determination of the configurations for positioning, clamping and support of a part. i.e. the selection of corresponding part faces and the selection of the appropriate fixture tools. The application of fixtures includes the assembly of fixtures (putting together fixture elements from a modular tool kit or using special designed fixture tools $[2,7,8]$ ) and the positioning, alignment and clamping of the fixture tools $[2,7,8]$ ) and the positioning, alignment and clamping of the
fixture on a pallet or a machine tool table, followed by the alignment and fixation of the workpiece in the fixture.

Positioning means, that the workpiece which has to be machined, has to have an accurate position and orientation with respect to the coordinate system of the machine tool, this to enable the machining of the part within the prescribed tolerances. The positioning procedure is prepared for in the fixture design phase, in which some of the faces of the workpiece which are accurate enough, are selected to act as positioning faces. The positioning faces serve as the reference points during the machining of the part.

The second phase takes place on the shop floor during the positioning and

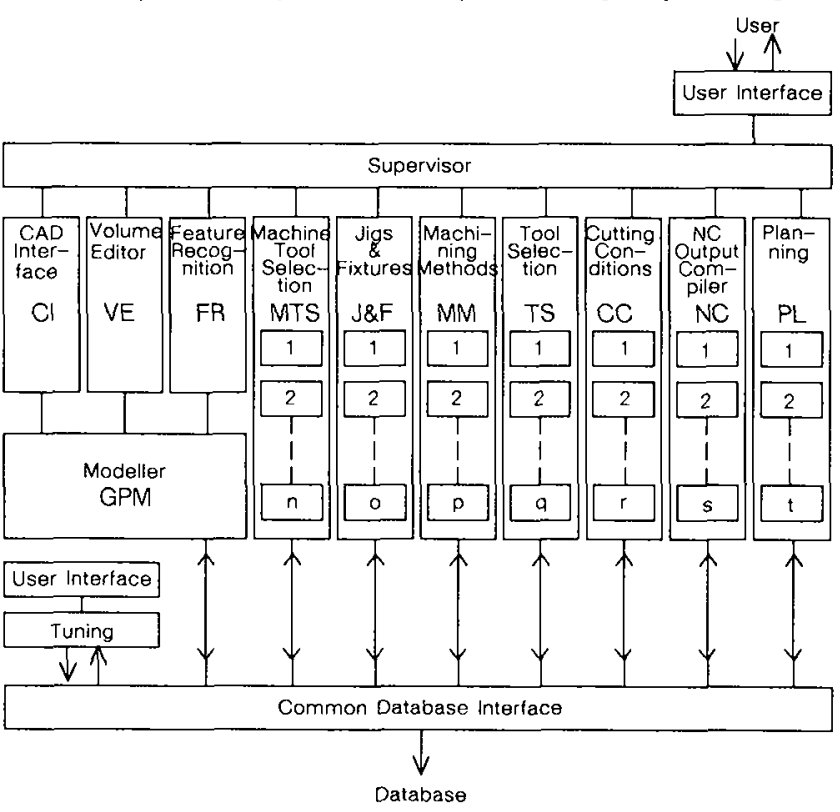

Figure 1. Schematic Representation of the PART system

alignment of the workpiece with the machine tool coordinate system.

2.2. accuracy considerations

The manufacturing accuracy of part elements depends on the path of the cutting tool relative to the workpiece. The resulting accuracy will be unsat isfactory when the part elements which have to be machined, are not in the prescribed position and orientation. The accuracy with which the part has to be positioned and aligned, is depending on the accuracr of the relations between the elements which have to be machined and other part elements, e.g. elements which have been machined in a previous set-up. This means, that the positioning accuracy is governed by the relation This means, that the positioning between elents. in other words, the selected set-ups determine the required positioning accu-
racy.

There are two more error-causing factors: the accuracy of the machining equipment [1] and the machining process itself. These factors are de- 
creasing the margins of accuracy between which the part has to be positioned.

\section{3. methods used in process planning and on the shop floor}

The process planner tries to limit the number of set-ups as much as possible for obvious economic reasons. More than one set-up is needed when the machining orientations of the part do not fit in one set-up [6]. For each set-up the process planner must select faces to position the part (positioning faces) and faces to support and clamp the part (suppor faces, clamping faces). Usually, the limitations in free space force the process planner to use the positioning faces simultaneously as support faces.

The positioning faces serve as geometrical references for the part ele ments which have to be machined. So the process planner has to find ments which haces which on the one hand are related to the elements which have to be machined and on the other are large enough to accept positioning
tools. If for a given set-up no adequate positioning faces can be found, tools. If for a given set-up no adequate positioning faces can be found
the process planner must rearrange the set-ups or he must decide to cre ate positioning faces, e.g. by machining them for that purpose in a previous set-up.

To position a part from the kinematic point of riew means constraining the six degrees of freedom of a free-moring body, i.e. three rotations and three translations. Applied to process planning this is translated into the so-called 3-2-1 constraining method:

3 positioning points, located in one plane (the 3-plane), constraining 2 rotations and 1 translation

2 positioning points, located in another plane (the 2-plane), perpendicular to the 3 -plane, constraining 1 rotation and 1 translation

1 positioning point, located in a third plane, perpendicular to both the 3-plane and the 2-plane, constraining one translation; see fig. 2.

The direction of the 3-plane is defined as perpendicular to the plane, i.e. the direction of $Y$. The directions of the 2-plane and the 1-plane successively are $X$ and $Z$.
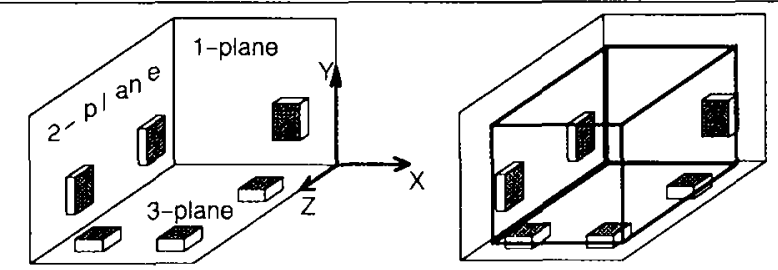

Figure 2. The 3 positioning planes and the 3-2-1 constraining method

For reasons of stability in the support of the part and the accuracy in the orientation of the part, the process planner selects by preference a relative large face of the part, this to locate the three positioning areas which compose the 3-plane, at a sufficient distance from each other. This approach is correct if three separate positioning faces exist on the fixture. However, often such a large face is placed directly on the machine tool table, resulting in unpredictable deflections of the part, caused by the insufficient flatness of the machine tool table (an overconstrained situation); see fig. 3. The application of a machine vice gives also a position-
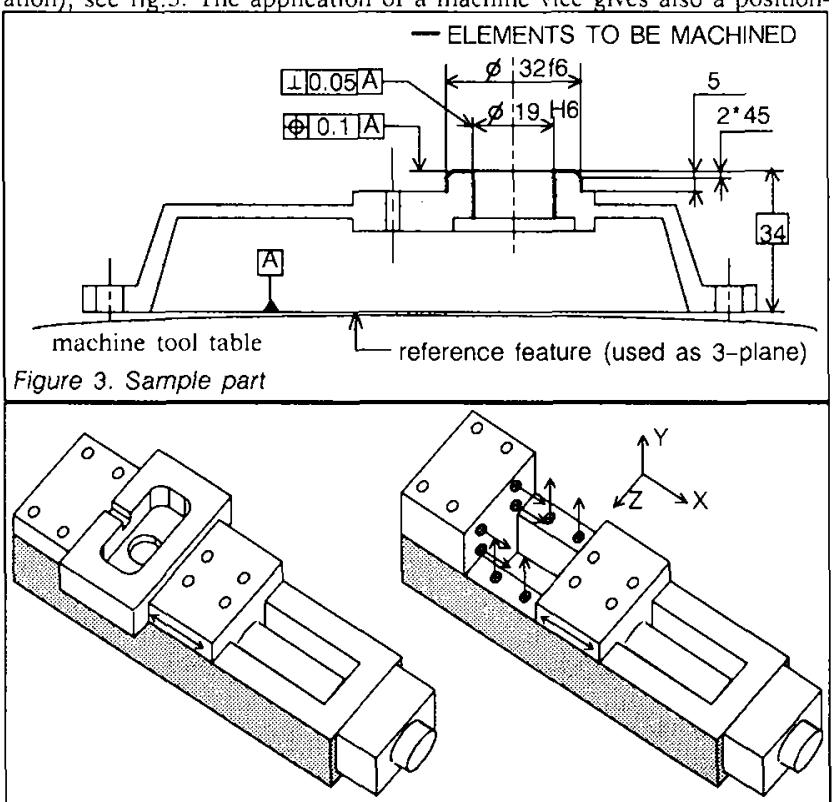

Figure 4. Positioning in a machine vice

ing configuration, which is kinematically incorrect; see fig.4. In this case. the part is overconstrained in two directions $(X . Y)$ and statically indeterminate in the remaining clirection $(Z)$. The workpiece is fixed in the $Z$-direction by means of friction forces, resulting from relatively high clamping forces which also can lead to part deflections. Consequently, this tvpe of fixturing equipment is only applicable for solid blanks and limited positioning requirements.

3. Design of an automatic planning procedure for part positioning 3.1. problem definition

In the workshop, the positioning of a workpiece for a given set-up consists of two steps:
1. placing (specific elements of) the workpiece on a prescribed $X, Y, Z$ position of the coordinate system of the machine tool

2. aligning (specific elements of) the workpiece with the $X, Y$ and $Z$ axes of the machine tool coordinate system

It is important to realize, that the possibility to compensate positioning errors with the aid of the machine tool controller is limited to translations coinciding with the machine tool axes. On the other hand if the workpiece is positioned with an alignment error, the workpiece has to be repligned by hand, since the CNC controller can not compensate for this. Positioning errors are the result of the positioning and the alignment on the machine tool but also of process planning where it is decided which faces are used as the positioning faces.

As for positioning, the process planner has to deal with the problems of selecting positioning faces, which (i) are most narrowly related to the elements of the part which have to be machined, (ii) provide a statically determinate lock up for the workpiece (iii) are accessible for the available positioning tools of the fixture, (iv) can create a stable workpiece position and (v) when necessary are large enough to fulfill the support function

\section{2. geometric approach}

The input of the process planning phase is the product model. Presently; in the part-manufacturing industry the two-dimensional drawing of a part still is the most widely used part representation. This representation is incomplete since the relations between the geometric elements in the different views represented in the drawing as such do not exist, but must be interpreted by the planner himself. However, an automated process planning system requires the input of a complete, unambiguously defined product model, i.e. a part representation generated by a solid modeller, containing all the geometric relations and tolerances as being required for the manufacturing process $[3,4,5]$

Instead of working with product models, in process planning it is better to deal with functional and recognizable part elements such as holes, pockets and plane faces, which are called features. In the present context a feature is a characteristic part element, defining a geometric shape, which is either specific for a machining process or which can be used for fixturing purposes. Subsequently, a feature can be described by its definition and the values of the corresponding parameters, e.g. the depth and the diameter of a hole. Features can be extracted from the product model using an automatic procedure $[10,11]$

Process planning can be executed only based on the data of the features instead of using the complete product model. This way of working is very efficient in the sense of data processing since the time-consuming processes of access to, and search in, a product model can be aroided.

As opposed to the geometric relations within features. the geometric relations between features are the only' important ones for fixturing, i.e.:

- the set-up selection must be based on the potential machining directions of features and the relations between the features which have to be machined [9]

- the selection of positioning faces for a given set-up must be based on the relations between the features which are already present and the features which have to be machined.

For positioning of parts faces are used which are selected from pre-selected features. The relations between the features already present and the features which have to be machined, which contain the smallest tolerances, provide the best candidate positioning faces. So, the selection of positioning faces can be based on the weighting of the tolerances of the relations between the two categories of features as mentioned above.

\section{3. possible strategies}

There are two different methods in use for positioning:

positioning and aligning the workpiece accurately on an exactly predefined location

aligning the workpiece accurately with the machine tool control axes

on a convenient location and adjusting the zero-coordinate of the machine tool controller to the selected zero-coordinate of the workpiece. As stated before, the machine tool controller can compensate for errors in position only; errors in the alignment of the workpiece have to be compensated by readjusting the orientation of the workpiece or the fixture.

However. there is a possibility to avoid the difficult and time-consuming positioning and alignment process by applying the "soft jaws" positioning method, as known from turning lathes, to machining centers. This practical method, from now on referred to as the "soft fixture" method, requires machining of the positioning faces of the fixture(s) after it has been assembled to the machine tool but prior to the positioning of the workpiece.

In the position faces selection process, two different accuracy aspects have to be dealt with:

the accuracy with respect to the kinematic fixation and the stability of the workpiece, related to the machining conditions

the accuracy with which the workpiece can be positioned and aligned in the ideal case

It may become evident that there are two different approaches possible for the planning and the realization of the fixture:

Method A: select some of the already present features as candidate positioning faces, considering in the first place favorite conditions for the kinematic solution and the stability of the workpiece. Next, check if the candidate faces are (i) geometrically narrowly related to the features which have to be machined, (ii) large enough to be used together with the available positioning tools.

Method B: select from the already present features those which geometrically are most narrowly related to the features which have to be ma. chined. Select from these features the candidate positioning faces coinciding with the three main positioning directions for subsequently the 3-point, 2-point and 1-point constraint. Next. the candidate faces are clecked for satisfying (i) the kinematic principles for constraining an object. (ii) the required stability of the part, (iii) the size requirements with respect to the available positioning tools. 
The following methods apply to the fixturing tasks on the shop floor. The methods 1 and 2 assume the presence of pallets, while the methods 3 and 4 deal with the assembly of fixtures on the machine tool table.

Method 1: the fixture is fixed on a pallet in a given position and orientation relative to the pallet coordinate system; subsequently the workpiece is fixed in the fixture

Method 2: the fixture is fixed on a pallet; the workpiece is assembled in the fixture in a given position and orientation relative to the pallet coordinate system

Method 3: the fixture is fixed on the machine tool table in a given position and orientation relative to the machine tool coordinate system, the workpiece is fixed in the fixture.

Method 4: the fixture is fixed on the machine tool table; the workpiece is assembled in the fixture in a given position and orientation relative to the machine tool.

3.4. Selection of a positioning method

As mentioned before, a part has to be positioned by means of the faces which are most narrowly related with the features which have to be machined. However, in actual practice it frequently occurs that potential positioning faces cannot be used because they are too small or not accessible for positioning tools. It also happens, that positioning faces have to perform a support function as well, in which case the size becomes important. with respect to the mechanical load

So, method $B$, which includes a systematic scanning of the appropriate relations, represents the best start, but in many cases it will not yield a

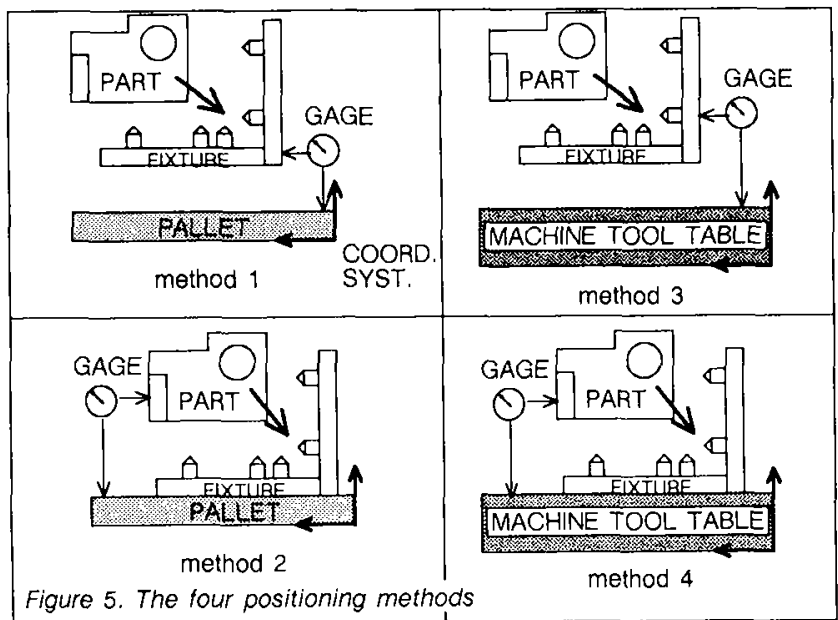

complete set (6) of usable positioning faces. The missing positioning faces have to be generated on the basis of method $A$

Starting to select positioning faces according to method $A$ is not wrong Starting to select positioning faces according to method $A$ is not wrong
but it means concentrating too much on the topology of the part instead of considering the geometric coherence of the features.

The best way of working is to start with method $B$ and, in case the number of positioning faces which can be found is insufficient, to continue with method $A$

Positioning and alignment of workpieces or fixtures direct on a machine tool table (fixturing methods 3 and 4) generally has to be avoided for economic reasons, but may be necessary in cases where high tolerances are involved In principle, the alignment of fixtures and workpieces on a machine tool table is more accurate, since no deviations are introduced by a pallet system. But, the use of adjustable positioning tools introduces again the danger of unintended readjustments during the exchange of workpieces and is therefore less reliable.

The soft fixture method provides positioning faces which are accurately positioned and aligned with the machine tool coordinate system. The method compensates for errors in fixture position and orientation. for errors due to the use of pallets and for systematic errors present in the machine tool itself. The method takes considerable less machine time than accurate fixture/workpiece positioning and alignment by hand since than accurate fixture/workpiece positioning and alignment by hand since Under the conditions that the process planning department is able to generate efficiently the working instructions and NC-programs for the machining of fixtures, the soft fixture method seems very attractive to apply, in particular in conjunction with the fixturing methods 3 and 4 .

From the foregoing, the next planning procedure has been derived.

1. for a given set-up, make an inventory of the geometric relations between the features which are already present and the features which have to be machined.

2. select the critical relations, considering the three principal directions of the part; the features already present and which take part in the relations are assigned as the reference features.

3. select positioning faces from the reference features, taking into account the size and focation of each reference feature as well as the kinematic principles and the stability of the part

4. when necessary, additional positioning faces can be selected from other features than the reference features. In that case, one has to take into account: (i) the accuracy of the relation between the new face and the features which have to be machined. (ii) the position and orientation of the face with respect to the other positioning faces and (iii) the size of the face

5 . if the number of positioning faces is still insufficient, an adaptation of the part is required; new acceptable positioning faces can be generated e.g. by assigning more accurate tolerances to relations containing initial, unusable candidate positioning faces. (In that case, these tolerances must actually be achieved during a previous machining opera- tion. Another possibility is to change the sequence of set-ups and to restart the selection of positioning faces.

4. description of the procedures for the selection of positioning, clamping and support faces

4.1. the procedure for the automatic selection of positioning faces To select the most critical relations between the features concerned, different types of tolerances have to be compared. For this, the different types of tolerances are converted into a so-called tolerance factor T.F The factor is based on the maximum allowable angle of misalignment of a toleranced feature (TOF) with respect to a reference feature (REF) which is determined by the free space as indicated in fig. 6.

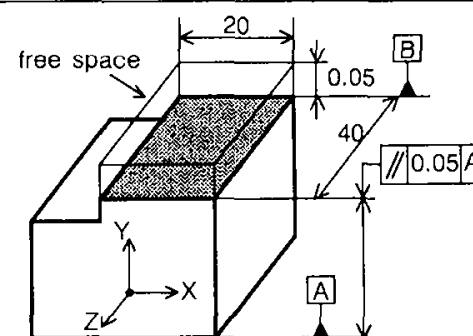

B: toleranced tace (TOF) A: reference face (REF)

relevant lengths:

$L X=20$

$L Z=40$

allowable misalignment of 8 $\mathrm{RX}=0.05 / 40$

$\mathrm{RZ}=0.05 / 20$

Figure 6. Allowable misalignment within a parallel tolerance

Only the alignment errors are considered, since the position errors can be compensated by the machine tool controller. The tolerance factor is calculated by dividing the tolerance value by the vectorial sum of the relevant lengths; see fig.7.

The tolerances of all the relations between the features are converted into tolerance factors; all the values of the T.F. are gathered in a so-called converted tolerance scheme (C.T.S.).

For each set-up the positioning faces are selected according to the following procedure:

generate the C.T S

2. select the three basic alignment reference features (BREF's)

3. select the directions for the three positioning planes

4. select the positioning faces from the BREF's

1. generate the C.T.S

As described in chapter 3.4., the selection of positioning faces is based on the selection of the most narrowly toleranced geometric relations between TOF's and REF's. Contrary to the set-up selection procedure, in the present context a REF is always a feature which already exists. The BREF's are selected from the REF's

The relations on which the C.T.S is based, can not always easily be found. The REF's can be (i) features which have been machined in a previous set-up, or (ii) features of a blank. The first ones may have rela-

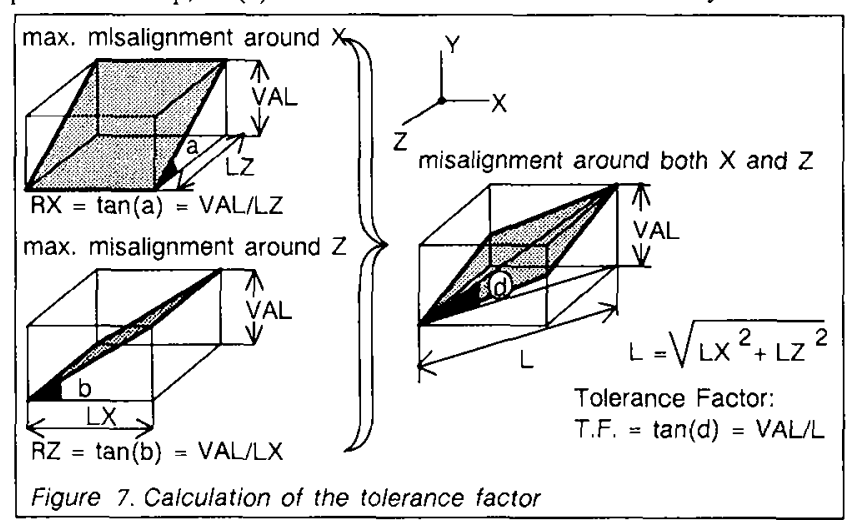

tions with the features which have to be machined; in that case the values for the C.T.S. can be calculated. However, relations between blank features and the features which have to be machined, are not always available, in which case they still have to be defined either explicitly or by default value.

2. Select the three basic alignment reference features

The C.T.S. represents the maximum allowable values of misalignment of the TOF's with respect to the REF's. To start with for each of the three principal axes the smallest value of either RX, RY and $R Z$ (see fig.8.) is determinated. The corresponding values represent the alignment conditions, which must be satisfied. The REF's in the corresponding three relations are the BREF's: in the example of fig. 8 the features 18,4 and 18 correspond with respectively the $X, Y$ and $Z$ direction.

\begin{tabular}{|c|c|c|c|c|c|c|c|c|c|c|c|c|}
\hline \multicolumn{2}{|c|}{ TOF } & \multicolumn{2}{|c|}{ REF } & \multicolumn{9}{|c|}{ TOLERANCE } \\
\hline \multirow[t]{2}{*}{ NR. } & \multirow[t]{2}{*}{ FO } & \multirow[t]{2}{*}{ NA. } & \multirow[t]{2}{*}{ FO } & \multirow[t]{2}{*}{ TYPE } & \multirow[t]{2}{*}{ VAL } & \multicolumn{3}{|c|}{ LENGTH } & \multicolumn{3}{|c|}{ MISALIGNMENT } & \multirow[t]{2}{*}{ T.F. } \\
\hline & & & & & & $L x$ & LY & $L Z$ & $\mathrm{RX}$ & RY & $R Z$ & \\
\hline 1 & Y & 18 & $-Y$ & $/ 1$ & 0.08 & 20 & & 10 & .008 & & .004 & .0036 \\
\hline 13 & $-x$ & 18 & $-Y$ & $\perp$ & 0.08 & & 10 & & & & .008 & .008 \\
\hline 7 & $z$ & 18 & $-\gamma$ & $\perp$ & 0.1 & & 40 & & .0025 & & & .0025 \\
\hline 12 & z & 4 & $z$ & $/ /$ & 0.09 & 10 & 20 & & .0045 & .009 & & .004 \\
\hline 17 & $-x$ & 18 & $-Y$ & $\perp$ & 0.07 & & 20 & & & & .0035 & .0035 \\
\hline 16 & $-z$ & 11 & $z$ & II & 0.1 & 10 & 20 & & .005 & .01 & & .0045 \\
\hline
\end{tabular}

Figure 8 . A converted tolerance scheme for positioning 
The T.F. in fig. 8 represents the value of:

$$
\operatorname{MIN}\left[\frac{V A L}{\sqrt{L X^{2}+L Z^{2}}} \cdot \frac{V A L}{\sqrt{L Y^{2}+L Z^{2}}} \cdot \frac{V A L}{\sqrt{L X^{2}+L Y^{2}}}\right]
$$

indicating the maximum allowable value of misalignment in any direction perpendicular to respectively the $Y, X$ and $Z$ axis.

3. Select the direction for the three positioning planes

Aligning a workpiece with two axes is considered more difficult than aligning with one axis only. The positioning faces of a 3-plane determine the workpiece alignment in two (principal) axes and the workpiece position on the third axis. Therefore, the 3-plane has to be selected by taking into account the maximum misalignment which is simultaneously allowed in two axes. Since the value of misalignment is expressed by the T.F., the 3-plane can be determined by selecting the relation with two alignment constraints, which contains the smallest T.F. The F.O. of the REF in volved represents the direction of the 3 -plane. In fig. 8 , the relations containing the features 18,4 and 11 determine the alignment in two axes; after comparing the T.F.'s, feature 18 is selected; the direction of the 3-plane becomes -Y. (N.B. the direction of the positioning plane is defined as perpendicular to the plane itself; see fig.2.)

felecting the direction of the 2-plane takes place by selecting the BREF Selecting the direction of the 2-plane takes place by selecting the BREF
corresponding with the remaining principal direction. In the example of fig. 8 the BREF of the Y-direction, i.e. feature 4 , is selected.

The direction of the 1-plane is perpendicular to the 3-plane and 2-plane which means two possible orientations of the plane. The direction canno be derived from the C.T.S. by considering the smallest allowable alignment error; the direction is selected depending on the available faces in one of the possible orientations. The selection of the 1-plane direction is not very important with respect to alignment, since the 1-plane does not take part in the alignment constraints and the positioning errors which are governed by the 1-plane, can be compensated.

4. Select the positioning faces

Starting with any of the three BREF's selected earlier, the next procedure is executed:

Select from the BREF all the faces of which the direction is perpendicular to the direction of misalignment for which the BREP has been selected. Subsequently, select the faces of which the area is large enough to accept Subsequently, select the faces of which the area is large enough to accept
a positioning tool. Areas of which the length and/or width exceed a proa positioning tool. Areas of which the length and/or width exceed a pro-
portion of the length and/or width of the whole part. are split up into two respectively four areas. Under the condition that the separated areas are large enough to accept a positioning tool, thes are considered as separate faces for the remainder part of the procedure.

Repeating this procedure brings up all the possible positioning faces which are directly and most accurately related to the TOF's. When the 3-2-1 constraining method cannot be satisfied with the aid of the now available faces, then additional REF's are looked for to supply additional positioning faces as to complete the positioning configuration in the re quired directions.

When still no complete 3-2-1 positioning configuration can be achieved, three other ways can be followed:

1. exchange the directions of the 3-,2- and 1-planes

2 . determine another sequence of set-ups

3. adapt the topology of the workpiece as to satisfy the positioning requirements

4.2. The procedure for the automatic selection of clamping faces

It is the function of the clamping tools to keep the workpiece in position and to maintain the orientation during machining. Clamping tools are part of the fixture and require adequate faces of the workpiece to act on (the clamping faces). Clamping means locking the workpiece between support and clamping tools. The support tools are applied to relieve the positioning faces from the clamping forces.

In traditional fixturing. the position tools almost always used to fulfill the support function as well. This is acceptable when (i) the spatial configuration of the positioning faces/tools ensures a stable workpiece position and (ii) the positioning faces are large and strong enough to withstand the clamping load. The consequences of the use of support tools with respect clamping load. The consequences of the use of support tools with resp

to the influence on the positioning accuracy is not discussed here.
The selection of clamping faces consists of the selection of support faces, followed by the selection of clamping faces.

The selection of the support faces

Procedure to be followed for all six 6 support faces:

1. select a positioning face (start with the 3-plane)

2. check the face on adequate size and strength. If satisfactory, then the positioning face will simultaneously be used as support face. If not satisfactory then continue:

3. select a separate support face as close as possible to the positioning

face and with the same orientation

4. check the support face for adequate size and strength.

The selection of the clamping faces

For each of the six support faces a matching clamping face is selected, located by preference opposite to the support face. The procedure goes as follows:

1. select a support face (start with the 3-plane)

2. select faces from the REF's of which the direction coincides with the direction of support; see fig. 9

3. select a face which is large enough to accept a clamping tool and which is located as much as possible overhead the support tool

4. when applicable. reduce the number of the clamping faces in the following was: when possible, different clamping forces are replaced by their centerload and the corresponding face is selected as clamping face. This procedure is rather straightforward if the location of the face. This procedure is rather straightforward if the location of the
centerload can be used as a clamping face; see fig.10. If not. an approximate face is looked for

Reducing the clamping faces leads to an increased deflection of the workpiece.
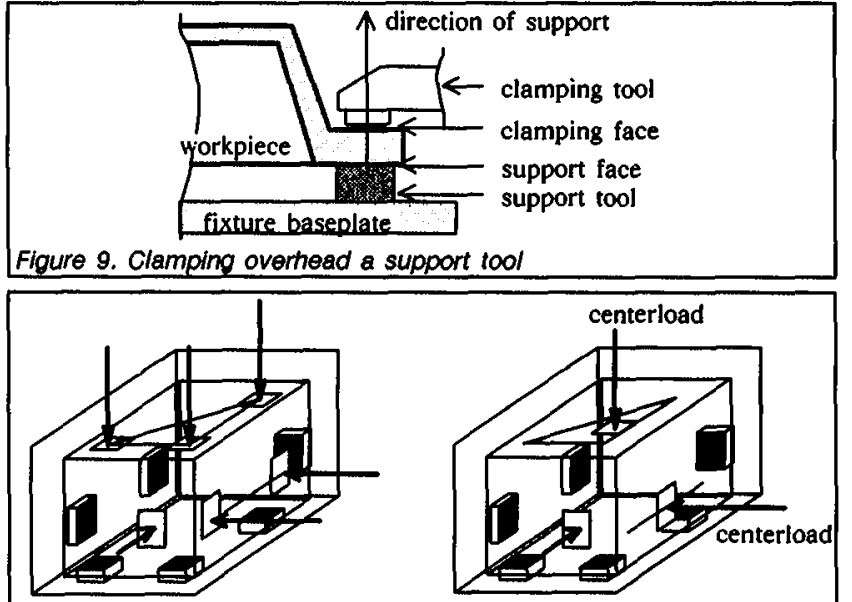

Figure 10. The reduction of clamping taces

4.4. The procedure for the selection of additional support faces.

Up till this phase in the planning procedure, the deflection of the workpiece is not considered. A workpiece is liable to deflect under its own weight and under machining and clamping loads. Methods to avoid deflection are (i) reducing the load by selecting a different machining method or different cutting conditions and (ii) applying support tools.

Reducing the load is economically not always acceptable (longer machining times), which emphasizes the possible application of additional support. The procedure follows:

1. calculating the locations at which maximum deflection occur

2. selecting support faces at or near these locations

The workpiece is analyzed for deflection. Since a product model is available, it is possible to examine the behavior of a simplified product model with the aid of a Finite Element Method (FEM) program. When during the design of a workpiece already a FEM analysis has been performed, the results may already be available.

The calculated maximum displacements are compared with the tolerance requirements. Potential locations for additional support are those places of which the displacement is larger than allowed by a tolerance.

For each of the potential support locations a support face is selected, which as much as possible is situated at or near the location of maximum displacement. The orientation of the support face must be equal to the direction of the displacement.

\section{Conclusions}

Computer aided process planning is only going to work if all planning tasks are represented in the system. With the implementation of the procedures for the selection of positioning, clamping and support faces, which links up with the previously implemented procedures for the selection of set-ups, another important chapter in process planning has been solved. Without this, the fixturing process would stay a serious impediment in the realization of integrated process planning systems. At present, the FIXES prototype contains the implemented routines for
the selection of set-ups and positioning faces and runs on $\mu$ VAX/VMS the selection of set-ups and positioning faces and runs on $\mu$ VAX/VMS
using $\mu$ VAX2000 and SiliconGraphics workstations. Future development is directed towards the implementation of the procedures for the selection of clamping and support faces as well as the automatic selection of fixture tools.

Acknowledgment

This research has been supported by the Netherlands Technology Foundation (STW).

References

1 Hocken,R.J.;1980: Machine tool accuracy, Technology of machine tools, vol.S, L.Livermore Nat.Lab.,Livermore, CA USA

2 Markus,A.;1987: Strategies for the automated generation of modular fixtures, working paper E/49/87, MTA/SZTAKI Univ. of Hungary, Budapest

3 Kimura,F.,Suzuki,H.,Inui,M.,Sata,T.;1988: A product modelling system for constructing intelligent CAD\&CAM systems, Robotics \& Comp.Integrated Manufacturing, vol.4, no.3/4, pp 483-489

4 Weill,R.et.al.;1988: Tolerancing for function, Annals of CIRP, vol. 37/2/1988

5 Weil,R.:1988: Integrating dimensioning and tolerancing in computer aided process planning, Robotics \& Comp.Integrated Manufacturing,
vol.4, no.1/2, pp 483-489

6 Ferreira,P.M.,Liu,C.R.;1988: Generation of workpiece orientations for machining using a rule-based system, Robotics \& Comp.Integrated Manufacturing, vol.4, no.3/4, pp 483-489

7 Grippo,P.M..Thompson,B.S.,Gandhi,M.V.;1988: A review of flexible fixturing systems for computer integrated manufacturing, Int. Journal of Comp. Integrated Manufacturing, vol.1, no.2. pp 124-135

8 Jiang,W.,Wang,Z.,Cai,Y.;1988: Computer-aided group fixture design, Annals of CIRP, vol.37/1/1988, pp 145-148

9 Boerma,J.R.,Kals,H.J.J.:1988: FIXES, a system for automatic selection of set-ups and design of features, Annails of CIRP, vol.37/1/1988, pp
$443-446$

10 Erve,A.H.van 't.Houten,F.J.A.M.van;1988: PART, a parallel approach to computer aided process planning, 4th Int. Conf. on Computer Aided
Engineering (CAPE). Edinburgh, Proceedings, pp 281-288, 1988

11 Erve, A.H.van ' $t$, : Generative computer aided process planning for part manufacturing. Ph.D. Thesis, University of Twente, 1988 